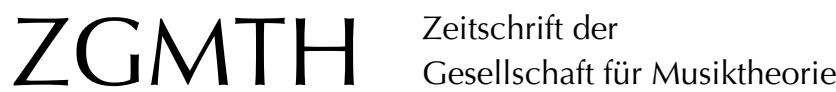

Moraitis, Andreas (2012): Nordic Conference on aural Disciplines in higher Music

Education - The Musical Ear: as a phenomenon, as a discipline, and in function. 10.12. Oktober 2012, Norwegian Academy of Music, Oslo. ZGMTH 9/2, 303-305. https://doi.org/10.31751/682

(C) 2012 Andreas Moraitis

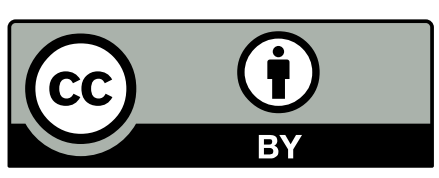

Dieser Text erscheint im Open Access und ist lizenziert unter einer Creative Commons Namensnennung 4.0 International Lizenz.

This is an open access article licensed under a

Creative Commons Attribution 4.0 International License.

veröffentlicht / first published: 15/03/2013

zuletzt geändert / last updated: 04/08/2014 


\section{Nordic Conference on aural Disciplines in higher Music Education - The Musical Ear: as a phenomenon, as a discipline, and in function, 10.-12. Oktober 2012, Norwegian Academy of Music, Oslo}

Nicht weniger als vierundzwanzig Jahre nach der ersten, 1988 in Kopenhagen abgehaltenen nordischen Konferenz zu Fragen des musikalischen Hörens im Kontext akademischer Musikausbildung wurde im Oktober 2012 der zweite Kongress dieser Art in Oslo ausgetragen. Im Fokus der von sechs Hochschullehrerinnen der Norwegian Academy of Music unter Leitung von Inger Elise Reitan organisierten Veranstaltung standen die Bereiche "Listening", »The Musical Ear and Performance« sowie "Aural Methodology». Mit John Sloboda (GB) und Gary S. Karpinski (USA) konnten zwei renommierte auswärtige Keynote-Speaker gewonnen werden, denen Erja Joukamo-Ampuja (FIN) und Frøydis Ree Wekre $(\mathrm{N})$ als Repräsentantinnen des skandinavischen Raums zur Seite standen.

John Sloboda thematisierte in seinem Referat das Verhältnis zwischen Musikern und ihrem Live-Publikum. Abnehmende Zuschauerzahlen im «klassischen Konzertbetrieb der letzten Jahrzehnte seien als Zeichen dafür zu werten, dass Aufgeschlossenheit gegenüber dem Repertoire und musikalische Kompetenz bei potenziellen Zuhörern nicht mehr als selbstverständlich vorausgesetzt werden könnten. Dementsprechend sei es notwendig, die jeweiligen Zielgruppen durch spezielle, konzertbegleitende Angebote zu schulen und zu motivieren, was am Beispiel mehrerer aktueller Projekte konkretisiert wurde. Inger Elise Reitan präsentierte eine Untersuchung zu Hörstrategien von Orchestermusikern im Vergleich zu denen eines Dirigenten. Der Gegensatz zwischen professioneller und nichtprofessioneller Rezeption wurde von Bengt Lundin (S) mit Bezug auf Musik zu Filmen Ingmar Bergmans herausgearbeitet, während
Reidar Bakke $(\mathrm{N})$ die Rolle natürlicher Geräusche und Klänge in Kompositionen jenseits einer stereotyp organisierten Musique concrete thematisierte. Gro Shetelig $(\mathrm{N})$, deren Moderationen des morgendlichen »Aural awakening « zu den inspirierenden Momenten der Konferenz gehörten, bot eine anschauliche Präsentation zu Fragen der Intonation von Sekundintervallen, die zu dem (freilich sehr allgemein gefassten) Begriff der $>$ Mikrotonalität in Beziehung gesetzt wurden. Ein Beitrag von Andreas Moraitis (D) über ein neuroakustisches Modell der Tonhöhen- und Grundtonerkennung sowie ein Bericht von Hannes Taljaard (ZA) über eine Studie zur Selbstbeobachtung von Musikern bildeten den Abschluss des ersten Tages.

Anregungen zur fachübergreifenden Diskussion gaben die beiden Hornistinnen Erja Joukamo-Ampuja und Frøydis Ree Wekre mit ihrer Keynote über die spezifischen Erfordernisse der Ausbildung von Blechbläsern. Schwerpunkte bildeten die Darstellung instrumentenbaulich bedingter Intonationsprobleme und die Dokumentation von Schwierigkeiten, welche sich beim Prima-vista-Spiel von Stimmen mit wechselnden Transpositionsintervallen ergeben. Dabei wurde die Notwendigkeit sinneren Hörens` besonders herausgestellt ein im Verlauf des Kongresses wiederholt angesprochener Punkt, der naturgemäß auch in Lotta llomäkis (FIN) Vortrag zum `Peer learning mittels instrumentaler Improvisation eine zentrale Rolle spielte. Leider wurde die Verfolgung des per Skype übertragenen Referats durch Verbindungsprobleme erschwert. Zwei von Aslaug Louise Slette $(\mathrm{N})$ und Guro Gravem Johansen $(\mathrm{N})$ vorgestellte Forschungsprojekte befassten sich mit Proben- und 
Übungskonzepten Studierender im Bereich der Kammermusik bzw. des Jazz. Die Bedeutung der musikalischen Analyse und ihres begrifflichen Instrumentariums für Gehörbildung und Aufführungspraxis stand im Mittelpunkt des Beitrags von Ingunn Fanavoll Øye $(\mathrm{N})$.

Für Irritationen sorgten die Ergebnisse einer von Anne Katrine Bergby $(\mathrm{N})$ präsentierten empirischen Untersuchung zum Verhältnis der Leistungsentwicklung von Studierenden in den Fächern Gehörbildung und musikalischer Praxis, da keinerlei Indiz für einen positiven Effekt der ersten auf die zweite Komponente gefunden werden konnte. Wünschenswert erschiene hier eine Erweiterung der Fragestellung, etwa im Hinblick auf die Motivation, das Trainingspensum oder die physischen Prädispositionen der Probanden. Victoria C. Jakhelln $(\mathrm{N})$ erörterte die Möglichkeiten der Verwendung technischer Hilfsmittel als Ergänzung zum konventionellen Gehörbildungsunterricht, wofür Carl-Axel Andersson (S) mit der Demonstration seiner gemeinsam mit Björn Roslund entwickelten Software MusicalEar später ein konkretes Beispiel liefern sollte. In einem separaten Beitrag diskutierte Andersson zunächst die Frage, inwieweit im Rahmen des (Jazz-) Ensemblespiels bewährte Kommunikationsmodelle auch für die propädeutische Hörerziehung nutzbar gemacht werden könnten. Inge Bjarke (DK) stellte ihren Lehrgang SNAPSHOT vor, der mit bemerkenswert geringem Aufwand den koordinierten Erwerb von Fähigkeiten im Lesen, Memorieren, Reproduzieren, Identifizieren und Transponieren musikalischer Phrasen ermöglicht. Eine stärkere Berücksichtigung fachspezifischer Bedürfnisse und die Vermeidung kontraproduktiven Leistungsdrucks empfahl Per-Olov Berndalen (S); statt die Lernenden durch das vorschnelle Einfordern von Antworten zu verunsichern, solle zunächst mittels kommentierter Improvisationen des Übungsleiters eine hinreichende Vertrautheit mit dem zu vermittelnden Stoff aufgebaut werden. Schließlich gab Carl Erik Kühl (DK) eine Einführung in seinen der Fehlererkennung gewidmeten Kurs Right Playing - Wrong Playing, für den eigens Aufnahmen originaler und veränderter Kompositionen verschiedener Stilepochen, Gattungen und Besetzungen eingespielt worden waren.

Gary S. Karpinsky ging in seinem engagierten Plädoyer für ein kontextuelles Hören von Ergebnissen wahrnehmungspsychologischer Forschung aus, deren Relevanz für das aurale Training er durch einige mit dem Publikum durchgeführte Experimente verdeutlichte. Insbesondere verwies er auf den Umstand, dass sich musikalische Strukturen nicht ohne Verluste auf ihre Komponenten reduzieren lassen, weswegen das am konkreten Werk orientierte Erarbeiten von Lerninhalten einer elementaristischen Methodik vorzuziehen sei. Offen blieb indes, ob in Anbetracht der oft knapp bemessenen Unterrichtszeiten die Vermittlung von Grundkenntnissen durch einen konsequent integrativen Ansatz lückenlos abgedeckt werden kann; auch wäre zu fragen, ob es angesichts der Fülle aktueller und potenzieller musikalischer Konstellationen möglich ist, auf die zwar wenig skunstgerechten<, dafür aber effektiven Verfahren der De- und Rekontextualisierung gänzlich zu verzichten.

Ein die Hochschulausbildung nicht direkt betreffendes, aber keineswegs unwichtiges Thema - die Entwicklung musikalischer Kompetenzen von Kindern im frühen Instrumentalunterricht - lag der von Hilde Synnøve Blix (N) präsentierten Studie zugrunde. Soila Jaakkola (FIN) stellte eine Untersuchung zur Bedeutung des Chorsingens für die Hörerziehung vor, während Dirkie C. Nell (ZA) über einen auf der 'Movable Dos-Methode beruhenden Ausbildungsgang für Studierende in den unteren Semestern und dessen Transfereffekte auf das Hörverhalten beim Instrumentalspiel berichtete. Franz Zaunschirm (A) erläuterte erneut sein auf einer Idee von Christian Möller beruhendes Konzept der 'Navigationsstimmes, wobei die zur Verfügung stehende Zeit vielleicht eine etwas ausführlichere Darstellung gestattet hätte. Die eingangs erörterte Frage kontextsensitiven Hörens wurde von Niels Eskild Johansen $(\mathrm{N})$ aufgegriffen, der eine eher liberale Position vertrat und dafür plädierte, den Lernenden bezüglich der zu wählenden Wege genügend Freiraum zu geben. So schlug er beispielsweise vor, ohne Schlüssel 
und Vorzeichen notierte Stimmen zu einer Klavierbegleitung singen zu lassen. Eine Reihe aufwendig gestalteter Unterrichtsmaterialien zur Harmonielehre stellt Johansen auf seiner Website (http://www.klassisk-harmoni.no) zur Verfügung.

Anders als bei den im deutschsprachigen Raum angebotenen Kongressen der letzten Jahre spielte die Erörterung spezifisch historischer Gesichtspunkte auf der Osloer Konferenz keine Rolle; selbst die bereits so intensiv diskutierte Thematik der mehrstimmigen Satzmodelle wurde kaum angesprochen. Angesichts der hohen Qualität der meisten Referate bedeutete dies keinen Nachteil; doch könnte hierin ein Potenzial für künftige Veranstaltungen liegen. Aufschlussreich und ausdrücklich zur Nachahmung zu empfehlen waren die von mehreren Vortragenden dargebotenen Videosequenzen aus der Unterrichtspraxis; überhaupt schien der Praxisbezug eine Leit- linie darzustellen, die sich durch fast alle Beiträge zog.

Ein erfreulicher Kontrast zu den im Kongressbetrieb sonst üblichen Verhältnissen ergab sich aus der durchweg sequenziellen Anordnung der Referate, die einen belastenden Sektionswechsel überflüssig machte. Beim gemeinsam eingenommenen Lunch, dem am zweiten Tag ein Überraschungsauftritt des jungen A-cappella-Ensembles Pitch vorausging, mehreren Networking breaks und einem um musikalische Elemente bereicherten Conference dinner bot sich den Teilnehmenden vielfältige Gelegenheit zum Erfahrungsaustausch; die ausgesprochen positive Atmosphäre, welche sich im Verlauf des Kongresses schnell aufgebaut hatte, dürfte Vielen die am Ende doch unvermeidliche Abreise schwer gemacht haben.

Andreas Moraitis 